\title{
TEXTILES FUNCIONALES COMO BARRERA DE PROTECCIÓN ANTE INFECCIONES ASOCIADAS A LA ATENCIÓN EN SALUD
}

\author{
$\triangle$ JENNIFFER ZAPATA-GIRALDO ${ }^{1}$ \\ LUZ E. BOTERO ${ }^{2}$ \\ MóNICA L MEJÍA ${ }^{3}$ \\ NELSON ESCOBAR-MORA ${ }^{4}$ \\ ISABEL ORTIZ-TRUJILLO ${ }^{5}$ \\ BEATRIZ J. GALEANO U. ${ }^{6}$ \\ LINA M. HOYOS $^{7}$ \\ DiAnA CuEsta ${ }^{8}$
}

\section{RESUMEN}

Los tejidos reforzados con nanoestructuras se ha convertido en un área de la investigación que busca contener la transmisión de microorganismos relacionados con las infecciones asociadas a la atención en salud, un problema de salud pública conlleva una alta carga de morbi-mortalidad en la población y pérdidas millonarias de recursos económicos. Las nanopartículas metálicas y óxido metálicas son agentes antimicrobianos que han cobrado importancia por su amplia

1 Ingeniera Biomédica, estudiante de Maestría en Ingeniería - Área Biomédica, Línea Microbiología de Sistemas, Grupo de Investigación Biología de Sistemas, Escuela de Ciencias de la Salud, Universidad Pontificia Bolivariana, Sede central Medellín.

2 Bacterióloga y laboratorista clínica, PhD Ciencias Médicas, docente titular Facultad de Medicina, Coordinadora Línea Microbiología de Sistemas, Grupo de Investigación Biología de Sistemas, Escuela de Ciencias de la Salud, Universidad Pontificia Bolivariana, Sede central Medellín.

3 Ingeniera Biomédica, estudiante de Maestría en Ingeniería - Área Biomédica, Línea Ingeniería Clínica, Grupo de Investigaciones en Bioingeniería, Universidad Pontificia Bolivariana, Sede central Medellín.

4 Ingeniero Mecánico, MSc Ingeniería. Coordinador de laboratorios de química y operaciones unitarias, Línea Ingeniería Clínica, Grupo de Investigaciones en Bioingeniería, Universidad Pontificia Bolivariana, Sede central Medellín.

5 Bióloga, PhD. Biología, docente titular Facultad Medicina. Coordinadora Grupo de Investigación Biología de Sistemas, Coordinadora Línea Mutagénesis y Epigenética Ambiental, Grupo de Investigación Biología de Sistemas, Escuela de Ciencias de la Salud, Universidad Pontificia Bolivariana, Sede central Medellín.

6 Ingeniera Mecánica, MSc Ingeniería. Coordinadora de Grupo de Investigaciones en Bioingeniería, Coordinadora Línea Ingeniería Clínica, Grupo de Investigaciones en Bioingeniería, Universidad Pontificia Bolivariana, Sede central Medellín.

7 Ingeniera Química, PhD. Ingeniería, docente investigador, Coordinadora Línea Nanosistemas, Grupo de Investigación Biología de Sistemas, Escuela de Ciencias de la Salud, Universidad Pontificia Bolivariana, Sede central Medellín.

8 Epidemiología, docente titular Facultad de Medicina, Coordinadora de Línea de Epidemiología, Grupo de Investigación en Salud Pública, Escuela de Ciencias de la Salud, Universidad Pontificia Bolivariana, Sede central Medellín.

Autor de correspondencia: Zapata Giraldo J. (Jenniffer): Universidad Pontificia Bolivariana, Sede central Medellín, circular 1, No.70-01, Medellín, Colombia. Tel.: (4) 4488 388, ext. 19333.

Correo electrónico: jenniffer.zapata@upb.edu.co
Historia del artículo:

Artículo recibido: 03-X-2017 / Aprobado: 23-IV-2017

Disponible online: 3 de mayo 2018

Discusión abierta hasta octubre de 2019 
aplicación. Esta revisión narrativa examina la evidencia científica de textiles funcionales con propiedades antimicrobianas como una estrategia para contener la transmisión de microorganismos relacionados con infecciones asociadas a la atención en salud, a partir del contexto de los textiles como fuente de contaminación e infección y los mecanismos antimicrobianos de las diferentes nanopartículas usadas como reforzantes para lograr un textil funcional. El desarrollo de la nanotecnología permite el progreso en diferentes campos de la ciencia y oportunidades en el ámbito de textiles funcionales.

PALABRAS CLAVE: Nanopartículas, Infecciones asociadas a la atención en salud, Control de la infección, Microbicidas, Textiles.

\section{FUNCTIONAL TEXTILES AS A PROTECTIVE BARRIER FOR HEALTH CARE ASSOCIATED INFECTIONS}

\section{ABSTRACT}

Fibers reinforced with nanostructures has become an area of research that seeks to contain the transmission of microorganisms connected with health care associated infections, a public health problem that carries high burden of morbidity and mortality in the population and millions of dollars lost. Metal and metal oxide nanoparticles are known to be antimicrobial agents with wide application. This narrative review aims to examine the scientific evidence of functional textiles with antimicrobial properties as a strategy to contain the transmission of microorganisms related with health care associated infections, starting from the context of textiles as a source of contamination and infection, and the antimicrobial mechanisms of the different nanoparticles used as reinforcers to achieve a functional textile. The development of nanotechnology will allow progress in different fields of science and opportunities in functional textiles.

KEYWORDS: Nanoparticles, Health care associated infections, Infection control, Microbicides, Textiles.

\section{TÊXTEIS FUNCIONAIS COMO BARREIRA À PROTEÇÃO CONTRA INFECÇÕES ASSOCIADAS AOS CUIDADOS DE SAÚDE}

\section{RESUMO}

O tecido reforçado nanoestrutural tornou-se uma área de pesquisa que busca conter a transmissão de microorganismos relacionados a infecções associadas com cuidados de saúde, um problema de saúde pública carrega uma carga elevada de morbidade e mortalidade na população e perdas milionários de recursos econômicos. As nanopartículas metálicas e os óxidos metálicos são agentes antimicrobianos que ganharam importância devido à sua ampla aplicação. Esta revisão narrativa examina a evidência científica de têxteis funcionais com propriedades antimicrobianas como estratégia para conter a transmissão de microorganismos relacionados a infecções associadas com cuidados de saúde, do contexto de têxteis como fonte de contaminação e infecção e mecanismos antimicrobianos de as diferentes nanopartículas utilizadas como reforçadores para atingir um tecido funcional. 0 desenvolvimento da nanotecnologia permite o progresso em diferentes campos da ciência e oportunidades no campo dos têxteis funcionais.

PALAVRAS-CHAVE: Nanopartículas, Infecções associadas com cuidados de saúde, Controle de infecção, Microbicidas, Têxteis. 


\section{INTRODUCCIÓN}

Las infecciones asociadas a la atención en salud (IAAS), se definen como "aquellas infecciones que el paciente adquiere mientras recibe tratamiento para alguna condición médica o quirúrgica y en quien la infección, no se había manifestado ni estaba en período de incubación en el momento del ingreso a la institución" (OMS, n.d.). Representan un importante problema sanitario y financiero en el mundo debido a la alta incidencia, a la morbilidad y mortalidad asociada y a la carga económica que imponen a pacientes, personal sanitario y sistemas de salud (PAHO, 2012).

En países en vía de desarrollo las IAAS ocurren en cada 6 a 20 de 100 pacientes mientras que en países desarrollados en 4-12\%. En Estados Unidos y Europa se estima que ocasionan entre 99 y 110 mil muertes anuales y 16 millones de días de estancia extra en hospitales (WHO, 2011)(Klevens et al., 2007)(Allegranzi et al., 2011). Según el Centro de Control de Enfermedades (CDC), los costos médicos anuales de infecciones en el ámbito hospitalario en Estados Unidos oscilaron entre \$ 28 y \$ 45 mil millones de dólares hacia el 2007. En América Latina, a pesar de que las IAAS son una causa importante de morbilidad y mortalidad, se desconoce la carga de enfermedad producida por este tipo de infecciones, aunque algunos países e instituciones se han aproximado a su cuantificación de manera independiente (PAHO, 2012).

La adquisición de las IAAS tiene en cuenta tres factores determinantes, la susceptibilidad del huésped, la virulencia del agente y el ambiente. Este último factor incluye el ambiente animado representado por el personal de salud, otros pacientes, y personas que acuden a la visita hospitalaria; mientras que el ambiente inanimado incluye el instrumental y equipos médicos, así como las superficies ambientales.

La contaminación de superficies del ambiente hospitalario varía de acuerdo con la humedad ambiental, la orientación vertical u horizontal de la superficie, la ventilación adecuada, la frecuen- cia de recambio de filtros de purificación del aire, el número de personas y tipo de actividad laboral que se realiza. Otros factores que contribuyen son la contaminación y crecimiento microbiano en textiles como cortinas, cubiertas para equipos, material de uso en el cuidado del paciente y del personal de salud como mantas, toallas, batas que están en contacto con fluidos corporales o partículas del ambiente (Sehulster et al., 2004). Una superficie contaminada no indica que causará una infección en un paciente o en el personal de la salud; para que ocurra es necesario completar la cadena de transmisión, es decir, que exista un hospedero susceptible, una puerta de entrada adecuada y un inóculo de microorganismo suficientemente virulento (Valderrama Beltran, Ariza Ayala \& Osorio Pinzon, 2010).

La aplicación de nanomateriales en la prevención de IAAS enfocada a las condiciones del ambiente hospitalario ha sido de reciente interés en la comunidad científica. Las nanopartículas (NP) se han utilizado para mejorar las propiedades de textiles existentes y para incorporar nuevas funciones, entre ellas, propiedades antimicrobianas. El uso de textiles funcionales en el ambiente hospitalario podría ser una barrera de protección de IAAS. Esta revisión narrativa pretende examinar la evidencia científica de textiles funcionales con propiedades antimicrobianas como una estrategia para contener la transmisión de microorganismos relacionados con IAAS.

\section{TEXTILES HOSPITALARIOS COMO FUENTE DE CONTAMINACIÓN}

Existe evidencia del rol que tienen los textiles que se usan en el área de la salud en la supervivencia y transmisión de patógenos en el ambiente hospitalario (Sattar et al., 2001)(Perry, Marshall \& Jones, 2001)(Treakle et al., 2009).

Las cortinas, que rodean la cama y brindan privacidad a los pacientes, pueden contaminarse por patógenos asociados a IAAS. Estudios realizados en dos hospitales de Estados Unidos revelaron la presencia de contaminación de las cortinas por 
patógenos, incluso resistentes a los antimicrobianos, como Enterococcus spp sensible y Enterococcus spp resistente a la vancomicina (VRE), Staphyloccocus aureus meticilino-sensible (MSSA), S. aureus meticilino-resistente (MRSA) y Clostridium difficile (Trillis et al., 2008)(Ohl et al., 2012). En Colombia, un estudio similar en un centro hospitalario de alta complejidad en Medellín, encontró que 30\% de las cortinas de servicios médico-quirúrgicos estaban contaminadas con MRSA, 35\% MSSA y 13\% con diferentes bacterias Gram negativas (Cataño et al., 2012). Existen tres posibles explicaciones para este hallazgo (i) contacto frecuente de los pacientes y personal de salud con las cortinas; (ii) falta de higiene de manos del personal de salud; (iii) poca frecuencia de cambio o lavado de las cortinas. Una estrategia que pudiera minimizar el impacto de esta contaminación es usar cortinas diseñadas con materiales con propiedades antimicrobianas.

Al igual que las cortinas y otros textiles de uso ambiental, la ropa que utiliza el personal de salud también es susceptible a contaminarse con patógenos; su desplazamiento permanente por los diferentes servicios hospitalarios representa un vehículo de transmisión a superficies, equipos u otro tipo de materiales y la diseminación de microorganismos incluso resistentes a múltiples fármacos. Frecuentemente, los trabajadores de la salud utilizan la misma ropa durante uno o dos días, tiempo suficiente para que se adquieran, retengan y transmitan (directa o indirectamente) patógenos epidemiológicamente importantes como MRSA y VRE a compañeros del trabajo (representando un riesgo laboral), a pacientes y al público en general (Callaghan, 1998).

Diversos estudios dan cuenta de la contaminación de textiles del personal de salud y de la ropa de los pacientes o de cama (Panagea et al., 2005). En un hospital de alta complejidad en Israel se encontró que $50 \%$ de la ropa (batas, ropa de cirugía o uniformes) del personal médico y de enfermería estaban contaminados por al menos un patógeno, como Acinetobacter spp, S. aureus (incluyendo cepas MRSA), Enterobacterias y Pseudomonas aeruginosa
(Wiener-Well et al., 2011). Otros estudios en Europa han reportado contaminación con microorganismos asociados con IAAS como C. difficile, VRE, Escherichia coli (Panagea et al., 2005).

La demostración de la relación entre la contaminación de textiles y la infección directa en pacientes o personal de salud ha sido limitada debido principalmente a la falta de análisis de los textiles cuando se presentan brotes infecciosos. Pocos estudios como los reportados sobre un brote de infecciones asociadas a catéter por Bacillus cereus en hospitales de Japón evidenciaron como principal fuente de la infección la contaminación de ropa de cama, las toallas de los pacientes (Sasahara et al., 2011) y del servicio de lavandería (Dohmae et al., 2008).

La ropa constituye una fuente de sustratos para el crecimiento bacteriano debido a que los microorganismos tienden a proliferar en ambientes ricos en proteínas, humedad y suciedad, condiciones fácilmente encontradas en los textiles que viste el personal de salud (Fijan \& Turk, 2012)(Boyce, 2007). Adicionalmente, la contaminación de textiles puede ser una fuente de reservorio como se evidenció en un análisis prospectivo durante cuatro semanas de 25 habitaciones de aislamiento de pacientes con MRSA en un hospital de alta complejidad de Irlanda; en más de la mitad de las muestras tanto en camas como colchones fueron positivas para MRSA y $70 \%$ de los aislamientos del ambiente eran idénticos o estaban estrechamente relacionados con los obtenidos en los pacientes que habían estado hospitalizados en las habitaciones (Sexton et al., 2006).

Aun cuando existen guías para la limpieza y descontaminación adecuada del ambiente hospitalario, los patógenos poseen mecanismos para adaptarse y sobrevivir durante periodos de tiempo prolongados (Esteves et al., 2016).

Los estudios reportados evidencian que la contaminación de superficies y textiles de la salud persiste posiblemente por fallas en la implementación o poca adherencia de las guías, incrementando con ello el riesgo de adquirir una IAAS (Sehulster $e t$ al., 2004)(Ling et al., 2015).

Revista EIA Rev.EIA.Esc.Ing.Antioq / Universidad ElA 


\section{NANOMATERIALES CON PROPIEDA- DES ANTIMICROBIANAS}

La nanotecnología ha emergido como un campo multidisciplinario de rápido crecimiento y con una variedad de aplicaciones. La manufactura de nuevos materiales en la escala nanométrica está ligada a sus prometedoras propiedades mecánicas, ópticas, eléctricas, físicas y químicas.

Las nanopartículas (NP) son clústeres de átomos cuyo tamaño en la escala nanométrica corresponde a $1 \times 10^{-9} \mathrm{~m}$; su origen es variable y pueden ser metálicas como las de plata $(\mathrm{Ag})$ y oro $(\mathrm{Au})$ u óxido metálicas como el óxido de zinc ( $\mathrm{ZnO}$ ) y dióxido de titanio $\left(\mathrm{TiO}_{2}\right)$. Entre las propiedades de las NP descritas se encuentran su efecto inmunomodulador, facilitan el transporte para liberación controlada de fármacos e importantes propiedades antimicrobianas. Es allí donde el uso de NP puede aplicarse como método de barrera o de limpieza y desinfección en la contención de la transmisión de las IAAS.

\section{A. Nanopartículas de plata (NP-Ag)}

Las NP-Ag se han convertido en las NP metálicas más estudiada en diferentes campos como biología, medicina, análisis químico, electrónica, y en la industria farmacéutica, textil y cosmética. En el campo de la medicina son utilizadas en forma de líquidos o aerosoles para la descontaminación y desinfección de espacios y para modificar superficies de varios materiales, en particular fibras o polímeros aplicados a ropa (Oćwieja et al., 2015).

Las NP-Ag no necesitan ser funcionalizadas con antimicrobianos para lograr su efecto, ellas ejercen su actividad antimicrobiana per se a través de liberación de iones $\mathrm{Ag}^{+}$que interactúan con la pared o membrana celular y otras estructuras internas de los microorganismos (Brown et al., 2012).

La actividad antimicrobiana de las NP-Ag depende de varios aspectos: el mecanismo de síntesis oxidativa que libera $\mathrm{Ag}^{+}$ofrece un efecto antimicrobiano que es inexistente cuando se obtiene NP por reducción. El tamaño tiene una relación inversa con el efecto antimicrobiano dado que se facilita el ingreso de las NP al citoplasma y ocasionan daños selectivamente estructurales. Finalmente, las formas que otorgan mayor área de superficie de contacto tienen a su vez mayor efecto antimicrobiano, así la forma triangular truncada supera a las esférica o tipo varilla. (Lok et al., 2007) (Pal, Tak \& Song, 2007) (Bera, Mandal \& Raj, 2014).

\section{Actividad Antibacteriana}

Diferentes estudios han demostrado que las NP-Ag inhiben el crecimiento bacteriano desde el primer contacto de la NP, con efecto bactericida o bacteriostático, inclusive ante las formas resistentes a los fármacos.

El mecanismo de acción descrito inicia desde el contacto con la pared o membrana celular, allí se unen a las partes cargadas negativamente de los grupos de proteínas que contienen disulfuro y fósforo creando poros por los que fluye el contenido citoplasmático al exterior de las células, disipando el gradiente $\mathrm{H}^{+}$de la pared o membrana y algunas veces causando la muerte celular bacteriana (Knetsch $\&$ Koole, 2011). El paso de iones $\mathrm{Ag}^{+}$al espacio intracelular produce efectos adicionales como la inhibición de los citocromos de la cadena transportadora de electrones, unión al ADN y ARN ocasionándole daño e inhibiendo la replicación del ADN, denaturación de la subunidad ribosomal 30S, activación de la producción de especies reactivas del oxígeno (ROS), apoptosis e inhibición de la síntesis de pared celular en las bacterias Gram positivas (Lara et al., 2009).

Kim et al. (Kim et al., 2007) reportaron que las NP-Ag inhibían el crecimiento de E. coli mientras que poseía un efecto medio contra $S$. aureus; estos hallazgos sugieren que la actividad antibacteriana de NP-Ag depende si la bacteria es Gram negativa o Gram positiva, debido probablemente a su diferencia en la composición de la pared celular. 


\section{Actividad Antifúngica}

Las NP-Ag inhiben el crecimiento de hongos relacionados con las IAAS. El mecanismo de acción descrito comienza con la acumulación de las NP-Ag alrededor de la membrana celular, inducción de la apoptosis a través de la exposición de fosfatidilserina, daño en el ADN, activación de las metacaspasas, disrupción de la integridad de la mitocondria, inducción de liberación de citocromo $\mathrm{C}$ e inhibición de formación de biopelículas según lo demostrado por Artunduanga et al. (Artunduaga Bonilla et al., 2015), Kim et al. (Kim et al., 2008)(Kim et al., 2009), Panácek et al. (Panácek et al., 2009), Hwang et al. (Hwang et al., 2012), Monteiro et al. (Monteiro et al., 2011) y Vazquez-Muñoz et al. (Vazquez-Muñoz, Avalos-Borja \& Castro-Longoria, 2014).

\section{Actividad Antiviral}

Las NP-Ag han demostrado ser agentes antivirales contra un espectro de virus como el de la Inmunodeficiencia Humana (VIH), Influenza y Herpes Simplex (HSV).

El mecanismo antiviral varía de acuerdo al virus. Contra el VIH, HSV-1, HSV-2 y el virus de Parainfluenza humana tipo 3 (HPIV-3) se produce una inhibición de la entrada de partículas virales a las células humanas, afectando la replicación viral (Lara et al., 2010) (Gaikwad et al., 2013). Contra la Influenza H3N2, las NP-Ag destruyen selectivamente la estructura viral uniéndose a la envoltura (Xiang et al., 2013). Es necesario futuros estudios que permitan dilucidar el mecanismo exacto.

\section{Actividad Antiparasitaria}

La evidencia de la actividad antiparasitaria es escaza con dos estudios publicados contra Setaria cervi y Echinococcus granulosus. El efecto escolicida se atribuye a la inducción de apoptosis a través de la generación de ROS y la interacción electrostática entre las NP-Ag cargadas positivamente y el parásito cargado negativamente, esto asociado a complejos orgánicos de la superficie (Saini et al., 2016)(Rahimi et al., 2015).

\section{b. Nanopartículas de oro (NP-Au)}

Las NP-Au poseen extensa aplicación en áreas como medicina, optoelectrónica y materiales compuestos debido a las propiedades que presenta, tales como potencial dieléctrico, no toxicidad y fácil funcionalización. Entre ellas se destacan supresión de tumores, estudios de farmacocinética, liberación controlada de fármacos y genes, ambientales, en industria de alimentos e industria espacial (Zhang et al., 2015).

La actividad antimicrobiana depende del tamaño, la forma, la concentración, las modificaciones en su superficie y los métodos de purificación en el proceso de síntesis.

\section{Actividad Antibacteriana}

La actividad antibacteriana de NP-Au ha sido controversial debido a resultados contradictorios en las publicaciones. Se ha reportado que las NP-Au no tienen efecto bactericida (Hernández-Sierra et al., 2008), o que a altas concentraciones se logra un débil resultado (Sreelakshmi et al., 2011). Mientras que algunos autores reportan que la concentración inhibitoria mínima para bacterias es independiente del tamaño (Azam et al., 2009)(Arshi et al., 2011), otros autores concluyen que la efectividad antibacteriana incrementa conjuntamente con el tamaño (Badwaik et al., 2012), y algunos muestran que la es inversa tanto al tamaño como a la concentración (Shamaila et al., 2016). La modificación de la superficie de las NP-Au es determinante al momento de evaluar la actividad antibacteriana. Varios estudios han usado diferentes moléculas con el fin de mejorar la efectividad antibacteriana, demostrando un efecto sinérgico contra bacterias sensibles y resistentes a los fármacos, al igual que bacterias productoras de biopelículas (Brown et al., 2012)(Ramasamy, Lee \& Lee, 2016) (Mu et al., 2016). Finalmente, utilizar un método de purificación de las NP-Au previene falsos resultados 
antibacterianos debido a la presencia de iones $\mathrm{Au}$ (III) o reactivos sin reaccionar en la fase de síntesis.

Los mecanismos relacionados con la actividad antibacteriana de NP-Au comienzan con las fuerzas electrostáticas presentes tanto en la NP como en la bacteria, las cuales permiten la atracción y posterior unión a la pared celular que modifica el potencial de membrana, disminuye los niveles de ATP e induce a la muerte celular (Jacobson et al., 2015) (Cui et al., 2012).

\section{Actividad Antifúngica}

Está basada en la interacción directa con las proteínas encargadas de la regulación del gradiente de potencial a través de la membrana celular, lo cual conlleva a un retardo en el crecimiento y finalmente la muerte (Ahmad et al., 2013)(Wani, Ahmad \& Manzoor, 2013). No se identificaron estudios que hayan evaluado la actividad antiviral y antiparasitaria de las NP-Au.

\section{c. Nanopartículas de dióxido de titanio (NP-TiO2)}

El dióxido de titanio $\left(\mathrm{TiO}_{2}\right)$ es un semiconductor tipo $\mathrm{n}$ con tres estructuras cristalinas: anatasa, rutilo y brookita. Tiene propiedades como estabilidad, durabilidad, efecto biocida y actividad fotocatalítica. Entre las aplicaciones se encuentran ambientales, energía, textiles, alimentos y cosméticos (Bogdan, Zarzyńska \& Pławińska-Czarnak, 2015).

La propiedad antimicrobiana de las $\mathrm{NP}^{-} \mathrm{TiO}_{2}$ se relaciona con su estructura cristalina anastasa, forma, tamaño y la necesidad de ser activadas con luz UV para alcanzar su efecto.

La actividad fotocatalítica que se da tras la radiación con UVB y UVA, las NP-TiO ${ }_{2}$ generan ROS en la forma de peróxido de hidrógeno $\left(\mathrm{H}_{2} \mathrm{O}_{2}\right)$ y radicales de hidroxilo $\left(\mathrm{OH}^{-}\right)$, por lo que oxidan compuestos orgánicos de la célula a dióxido de carbono $\left(\mathrm{CO}_{2}\right)$ y agua $\left(\mathrm{H}_{2} \mathrm{O}\right)(\mathrm{Fu}$, Vary \& Lin, 2005)(Banerjee et al., 2006).

\section{Actividad Antibacteriana}

El daño ocasionado por las ROS en la integridad de las paredes y membranas celulares comprometen la semipermeabilidad de la membrana e interfieren con la fosforilación oxidativa ocasionando la muerte de la bacteria (Carré et al., 2014).

Por otra parte, Roy et al. (Aashis S. Roy, 2010) evaluaron el efecto de $\mathrm{NP}^{-T i O}{ }_{2}$ funcionalizadas con diferentes antibióticos contra $S$. aureus meticilinoresistente y encontraron que las $\mathrm{NP}^{-\mathrm{TiO}_{2}}$ mejoraron el efecto antimicrobiano de los antibióticos beta-lactámicos, cefalosporinas, aminoglucósidos, glicopéptidos, macrólidos, lincosamidas y tetraciclina contra MRSA. En otro experimento, se observó una disminución de la resistencia antimicrobiana de MRSA contra varios de los antibióticos con la presencia de $\mathrm{NP}^{-\mathrm{TiO}_{2}}$.

\section{Actividad Antifúngica}

Las $\mathrm{NP}^{-\mathrm{TiO}_{2}}$ poseen actividad antifúngica asociada con la producción de ROS a través de la propiedad de fotocatálisis. Thabet et al. (Thabet et al., 2014) evaluaron esta propiedad contra Saccharomyces cerevisiae, encontrando que la generación de ROS y la acumulación del mismo a nivel de la membrana celular son los encargados de la propiedad antifúngica. Por otro lado, diferentes estudios han evaluado $\mathrm{NP}^{-T i O}{ }_{2}$ contra $C$. albicans encontrando actividad antifúngica (Perelshtein et al., 2012)(Xiao et al., 2014).

\section{Actividad Antiviral}

Las $\mathrm{NP}_{-} \mathrm{TiO}_{2}$ han mostrado tener propiedades antivirales contra virus sin dilucidar el mecanismo. Jiang et al. (Jiang et al., 2009) y Cui et al. (Cui et al., 2010) observaron que las $\mathrm{NP}_{\mathrm{TiO}}$ en su fase anatasa combinada con $\mathrm{Cu}^{+2}$ tienen un efecto virucida contra el virus de la Influenza aviar H9N2 bajo una radiación a $365 \mathrm{~nm}$.

\section{Actividad Antiparasitaria}

No se encontraron publicaciones de la valoración de la actividad únicamente con $\mathrm{NP}^{-\mathrm{TiO}_{2}}$. 
Allahverdiyev et al. (Allahverdiyev et al., 2013) observaron el efecto antiparasitario a partir de una combinación de $\mathrm{NP}_{-} \mathrm{TiO}_{2}$ y NP-Ag en dos especies de Leishmania, esta mezcla tuvo la capacidad de inhibir propiedades biológicas como viabilidad, actividad metabólica y supervivencia dentro de las células hospederas. Adicionalmente, se observó el doble del efecto antiparasitario cuando las nanopartículas fueron irradiadas con luz visible.

\section{d. Nanopartículas de óxido de zinc} (ZnO)

El óxido de zinc es un semiconductor tipo $n$ con excelentes propiedades eléctricas y ópticas, así como una alta estabilidad química, baja toxicidad y fotocatálisis (Karunakaran, Rajeswari \& Gomathisankar, 2011)(Ramani et al., 2013).

Las $\mathrm{NP}-\mathrm{ZnO}$ al igual que el $\mathrm{NP}^{-\mathrm{TiO}_{2}}$ mejoran su actividad antimicrobiana cuando son irradiadas con UVA debido a las propiedades fotocataliticas que se dan por la conformación de pares electrón-hueco, que inducen la producción de ROS (Karunakaran, Rajeswari \& Gomathisankar, 2011)(Dodd et al., 2006).

\section{Actividad Antibacteriana}

El mecanismo de acción del efecto antibacteriano todavía es motivo de estudio, sin embargo se conoce que la actividad antibacterial de las NP-ZnO depende de la concentración y el tamaño de la nanopartícula, el efecto bactericida aumenta a medida que disminuye el tamaño de las partículas (Huang et al., 2008)(Reddy et al., 2014)(Padmavathy \& Vijayaraghavan, 2008). Emami-Karvani et al. (Zarrindokht Emami-Karvani, 2012) encontraron que con la disminución de tamaño de partícula y mayor concentración se aumentó la propiedad antibacteriana contra bacterias Gram negativas (E. coli) y Gram positivas (S. aureus).

Se han propuesto diferentes mecanismos que influencian el daño bacteriano (Zhang et al., 2008):
- $\quad$ Producción de ROS a partir de iones Zn2+ y peróxido de hidrógeno (H2O2).

- Acumulación de nanopartículas en la superficie de las bacterias y generación de fuerzas electrostáticas con la pared bacteriana. Xie et al. (Xie et al., 2011) evaluaron la actividad antibacteriana contra Campylobacter jejuni. Se encontró que el mecanismo de acción se relacionó con cambios morfológicos de la bacteria (ruptura de la membrana celular) e incremento en la expresión de genes para estrés oxidativo.

- Destrucción de los lípidos y proteínas de la pared celular bacteriana incrementando la permeabilidad, la internalización de las nanopartículas y cambios en la estructura celular.

- Interrupción del transporte de electrones transmembranales.

Azam et al. (Azam et al., 2012) reportaron una comparación de actividad antimicrobiana entre nanopartículas de $\mathrm{ZnO}, \mathrm{CuO}, \mathrm{y} \mathrm{Fe}_{2} \mathrm{O}_{3}$ en bacterias tanto Gram positivas como Gram negativas. De acuerdo a sus resultados, las nanopartículas con mayor efecto bactericida fueron las de $\mathrm{ZnO}$, mientras que las nanopartículas de $\mathrm{Fe}_{2} \mathrm{O}_{3}$ exhibieron los menores efectos.

\section{Actividad Antifúngica}

Las NP-ZnO poseen actividad antifúngica según Sharma et al. (Sharma et al., 2010), Kairyte et al. (Kairyte, Kadys \& Luksiene, 2013) y Gondal et al. (Gondal et al., 2012). Esta propiedad se relaciona con la capacidad fotocatalítica presente en las NPZnO y la producción de ROS, las cuales interactúan con la membrana celular de los hongos.

\section{Actividad Antiviral}

Las NP-ZnO han demostrado capacidad antiviral contra los virus de HSV-1 y HSV-2. Mishra et al. (Mishra et al., 2011) y Antoine et al. (Antoine et al., 2012) diseñaron micro-nanoestructuras de $\mathrm{ZnO}$ denominadas tretápodos que demostraron ser capaces de inhibir la entrada del virus a la célula hospede- 
ra, y el potencial de parar la diseminación del virus a lo largo de las células infectadas. El mecanismo detrás de la neutralización del HSV-2 se relaciona con la unión de las NP-ZnO con el virión de HSV-2. Adicionalmente, gracias a la irradiación con luz UVA se obtuvo aún más inhibición del virus.

\section{Actividad Antiparasitaria}

De otro lado, se han evidenciado propiedades antiparasitarias contra formas de Leishmania major. Delavari et al. (Delavari et al., 2014) observaron que al someter formas de promastigotes de Leishmania major bajo diferentes concentraciones de la NP-ZnO, hubo una reducción del parásito debido a apoptosis y necrosis. A las 72 horas de incubación de NP-ZnO con 120 нg/ml se logró una disminución del 93,76\% en la viabilidad del parásito.

En la Tabla 1 se resumen los principales mecanismos antimicrobianos de las nanopartículas.
4. TEXTILES FUNCIONALES:

\section{INNOVACIÓN EN PROPIEDADES}

\section{ANTIMICROBIANAS A TRAVÉS DE NANOPARTÍCULAS}

El uso de textiles antimicrobianos en entornos clínicos se asocia a nuevas medidas preventivas que permitan reducir la carga microbiana disponible, disminuyéndose el riesgo de adquirir IAAS (Borkow \& Gabbay, 2008) (Shahid-ul-Islam, Butola \& Mohammad, 2016). Gracias a los múltiples mecanismos de acción antimicrobiano resumidos en el apartado 3, la industria textil ha incorporado nanopartículas con la finalidad de otorgar a las fibras, características físicas y químicas adicionales, como antimicrobiano, bloqueo de UV, y autolimpieza, convirtiéndoles en textiles funcionales (Álvarez-Paino, Muñoz-Bonilla \& Fernández-García, 2017)(Montazer \& Maali Amiri, 2014).

TABLA 1. PRINCIPALES MECANISMOS ANTIMICROBIANOS DE LAS NANOPARTÍCULAS METÁLICAS-ÓXIDO METÁLICAS

\begin{tabular}{|c|c|c|c|c|}
\hline Mecanismo & Antibacteriano & Antifúngico & Antiviral & Antiparasitario \\
\hline $\begin{array}{l}\text { Liberación de iones metálicos e interacción de la } \\
\text { NP con la pared o membrana celular y estructuras } \\
\text { internas de microorganismos }\end{array}$ & $\begin{array}{l}\mathrm{Ag} \\
\mathrm{ZnO}\end{array}$ & $\begin{array}{l}\mathrm{Ag} \\
\mathrm{ZnO}\end{array}$ & - & $\begin{array}{l}\mathrm{Ag} \\
\mathrm{ZnO}\end{array}$ \\
\hline $\begin{array}{l}\text { Acumulación de las nanopartículas alrededor de } \\
\text { la pared y/o membrana celular }\end{array}$ & $\begin{array}{l}\mathrm{Ag} \\
\mathrm{ZnO} \\
\mathrm{Au}\end{array}$ & $\begin{array}{l}\mathrm{Ag} \\
\mathrm{ZnO}\end{array}$ & - & $\begin{array}{l}\mathrm{Ag} \\
\mathrm{ZnO}\end{array}$ \\
\hline $\begin{array}{l}\text { Interacción electrostática entre la nanopartícula y } \\
\text { el microorganismo }\end{array}$ & $\begin{array}{l}\mathrm{Ag} \\
\mathrm{ZnO} \\
\mathrm{Au}\end{array}$ & $\begin{array}{l}\mathrm{Ag} \\
\mathrm{ZnO} \\
\mathrm{TiO}_{2}\end{array}$ & - & - \\
\hline $\begin{array}{l}\text { Destrucción de los lípidos y proteínas de la pared } \\
\text { celular }\end{array}$ & $\begin{array}{l}\mathrm{Ag} \\
\mathrm{ZnO}\end{array}$ & - & - & - \\
\hline $\begin{array}{l}\text { Interrupción del transporte de electrones } \\
\text { transmembranales }\end{array}$ & $\begin{array}{c}\mathrm{Ag} \\
\mathrm{ZnO}\end{array}$ & - & - & - \\
\hline $\begin{array}{l}\text { Inducción de apoptosis a través de la generación } \\
\text { de ROS }\end{array}$ & $\begin{array}{l}\mathrm{Ag} \\
\mathrm{ZnO} \\
\mathrm{TiO}_{2}\end{array}$ & $\begin{array}{l}\mathrm{Ag} \\
\mathrm{ZnO} \\
\mathrm{TiO}_{2}\end{array}$ & - & $\begin{array}{l}\mathrm{Ag} \\
\mathrm{ZnO} \\
\mathrm{TiO}_{2}\end{array}$ \\
\hline Inhibición del ingreso a la célula del hospedero & - & - & $\mathrm{Ag}$ & - \\
\hline
\end{tabular}


Los textiles de uso hospitalario deben estar diseñados con materiales no porosos y con texturas regulares que faciliten la limpieza y prevengan el atrapamiento de polvo, deben resistir al proceso de limpieza y desinfección que se utiliza en servicios de salud o contar con un proceso de desinfección propio (Damani, 2012).

Diferentes estudios han evaluado las propiedades antimicrobianas de textiles reforzados con diferentes nanopartículas. Pollini et al. (Pollini et al., 2009), Perera et al. (Perera et al., 2013), Eremenko et. al. (Eremenko et al., 2016) y Balakumaran et al. (Balakumaran et al., 2016), incorporaron NP-Ag con diferentes métodos de síntesis a fibras de algodón para evaluar las propiedades antimicrobianas contra bacterias y hongos. Sus hallazgos evidenciaron que únicamente la síntesis in situ permitía la formación de NP-Ag distribuidas uniformemente en las fibras y con actividad microbicida la cual se mantenía después del lavado, mientras que con la síntesis ex situ los efectos fueron menores. Por otro lado, Np$\mathrm{TiO}_{2}$ y NP-TiO 2 /Ag han sido exploradas en los trabajos de Dastjerdi et al. (Dastjerdi et al., 2010) y Li et. al. (Li et al., 2017). Se evaluó la propiedad antibacteriana obteniendo más de un $99 \%$ de eficiencia contra S. aureus y E. coli.

Los textiles reforzados con NP-ZnO se han estudiado por Petkova et. al. (Petkova et al., 2016), Thi et. al. (Tran Thi \& Lee, 2017), Shaheen et. al. (Shaheen et al., 2016) y Uğur et. al. (Uğur et al., 2010). En todos los estudios se observa una disminución de unidades formadores de colonias de microorganismos, que varían entre bacterias y hongos, además de un efecto antimicrobiano prolongado inclusive después de varios lavados industriales.

Por otro lado, Schweizer et al. (Schweizer et al., 2012) y Kotsanas et al. (Kotsanas et al., 2014) desarrollaron estudios clínico en ciertas áreas hospitalarias para evaluar cortinas con NP-Ag propiedades antimicrobianas. Se encontró que el textil con partículas de plata duraba siete días más antes de tener la primera contaminación bacteriana comparado con la cortina estándar, lo cual hace posible dismi- nuir la transmisión de patógenos (Schweizer et al., 2012). Adicionalmente, se encontró que las cortinas con NP-Ag tuvieron actividad antimicrobiana contra 14 patógenos bacterianos, que incluían Clostridium difficile, y al hacer una evaluación costo-efectiva se demostró el uso benéfico de estas cortinas en las unidades de cuidados intensivos (Kotsanas et al., 2014).

Los textiles funcionales que incluyen en su estructura nanopartículas con efectos antimicrobianos tiene propiedades hidrofóbicas por lo que requieren menor frecuencia de lavado, y potencialmente un beneficio ambiental por la reducción de agentes químicos en el cuidado del textil, consumo de agua y energía (Palza, 2015)(Windler, Height \& Nowack, 2013)(Sarkar et al., 2012).

\section{CONCLUSIONES}

Las capacidades que poseen algunos patógenos de persistir en el ambiente hospitalario por periodos de tiempo prolongados y contaminar materiales presentes en las habitaciones de pacientes colonizados, representan un riesgo inminente de generar infecciones asociadas a la atención en salud. Estos microorganismos pueden ser transmitidos a través de las manos del personal de la salud, quienes se convierten en vehículos de transmisión para los pacientes y demás personal de la salud, personas relacionadas con el paciente u otras áreas hospitalarias.

Las instituciones prestadoras de servicios de salud están obligadas a garantizar las medidas necesarias para prevenir, controlar y en lo posible erradicar la propagación de IAAS desde los posibles medios de transmisión, entre ellos los fómites y textiles.

Las nanopartículas tienen un gran potencial como reforzantes en el área textil, debido a que confieren propiedades antimicrobianas. La incorporación de estas nanopartículas en los textiles que se usan en el área de la salud permitiría nuevos tipos de prevención contra la transmisión de IAAS.

Los textiles funcionales para uso hospitalario deben así tener características clave como, propiedades antimicrobianas contra un amplio espectro 
de microorganismos, incluso contra aquellos resistentes a los antibióticos, relacionados con IAAS proporcionado ambientes seguros para los humanos.

\section{AGRADECIMIENTOS}

A la Universidad Pontificia Bolivariana por la financiación a través de la convocatoria UPB INNOVA 2015, con radicado ante el CIDI 438B-08 / 15-65. A Colciencias por la financiación a través de la convocatoria Ciencia, Tecnología e Innovación en Salud 711-2015, bajo el contrato número 121071149742.

\section{REFERENCIAS}

Aashis S. Roy, A.P. (2010) Effect of Nano - Titanium Dioxide with Different Antibiotics against Methicillin- Resistant Staphylococcus Aureus. Journal of Biomaterials and Nanobiotechnology. [Online] 1 (1), 37. Available from: doi:10.4236/jbnb.2010.11005.

Ahmad, T., Wani, I.A., Lone, I.H., Ganguly, A., et al. (2013) Antifungal activity of gold nanoparticles prepared by solvothermal method. Materials Research Bulletin. [Online] 48 (1), 12-20. Available from: doi:10.1016/j.materresbull.2012.09.069.

Allahverdiyev, A.M., Abamor, E.S., Bagirova, M., Baydar, S.Y., et al. (2013) Investigation of antileishmanial activities of Tio2@Ag nanoparticles on biological properties of L. tropica and L. infantum parasites, in vitro. Experimental Parasitology. [Online] 135 (1), 55-63. Available from: doi:10.1016/j.exppara.2013.06.001.

Allegranzi, B., Bagheri Nejad, S., Combescure, C., Graafmans, W., et al. (2011) Burden of endemic healthcare-associated infection in developing countries: systematic review and meta-analysis. Lancet (London, England). [Online] 377 (9761), 228-241. Available from: doi:10.1016/S0140-6736(10)61458-4.

Álvarez-Paino, M., Muñoz-Bonilla, A. \& Fernández-García, M. (2017) Antimicrobial Polymers in the NanoWorld. Nanomaterials (Basel, Switzerland). [Online] 7 (2). Available from: doi:10.3390/nano7020048.

Antoine, T.E., Mishra, Y.K., Trigilio, J., Tiwari, V., et al. (2012) Prophylactic, therapeutic and neutralizing effects of zinc oxide tetrapod structures against herpes simplex virus type- 2 infection. Antiviral Re- search. [Online] 96 (3), 363-375. Available from: doi:10.1016/j.antiviral.2012.09.020.

Arshi, N., Ahmed, F., Kumar, S., Anwar, M.S., et al. (2011) Microwave assisted synthesis of gold nanoparticles and their antibacterial activity against Escherichia coli (E. coli). Current Applied Physics. [Online] 11 (1, Supplement), S360-S363. Available from: doi:10.1016/j.cap.2010.11.102.

Artunduaga Bonilla, J.J., Paredes Guerrero, D.J., Sánchez Suárez, C.I., Ortiz López, C.C., et al. (2015) In vitro antifungal activity of silver nanoparticles against fluconazole-resistant Candida species. World Journal of Microbiology \& Biotechnology. [Online] 31 (11), 1801-1809. Available from: doi:10.1007/ s11274-015-1933-z.

Azam, A., Ahmed, A.S., Oves, M., Khan, M.S., et al. (2012) Antimicrobial activity of metal oxide nanoparticles against Gram-positive and Gram-negative bacteria: A comparative study. International Journal of Nanomedicine. [Online] 7, 6003-6009. Available from: doi:10.2147/IJN.S35347.

Azam, A., Ahmed, F., Arshi, N., Chaman, M., et al. (2009) One step synthesis and characterization of gold nanoparticles and their antibacterial activities against E. coli (ATCC 25922 strain). Int J Theor Appl Sci. 1 (2), 1-4.

Badwaik, V.D., Vangala, L.M., Pender, D.S., Willis, C.B., et al. (2012) Size-dependent antimicrobial properties of sugar-encapsulated gold nanoparticles synthesized by a green method. Nanoscale Research Letters. [Online] 7 (1), 623. Available from: doi:10.1186/1556276X-7-623.

Balakumaran, M.D., Ramachandran, R., Jagadeeswari, S. \& Kalaichelvan, P.T. (2016) In vitro biological properties and characterization of nanosilver coated cotton fabrics - An application for antimicrobial textile finishing. International Biodeterioration \& Biodegradation. [Online] 107, 48-55. Available from: doi:10.1016/j.ibiod.2015.11.011.

Banerjee, S., Gopal, J., Muraleedharan, P., Tyagi, A.K., et al. (2006) Physics and chemistry of photocatalytic titanium dioxide: visualization of bactericidal activity using atomic force microscopy. Current Science. 90 (10), 1378-1383.

Bera, R.K., Mandal, S.M. \& Raj, C.R. (2014) Antimicrobial activity of fluorescent Ag nanoparticles. Letters in 
Applied Microbiology. [Online] 58 (6), 520-526. Available from: doi:10.1111/lam.12222.

Bogdan, J., Zarzyńska, J. \& Pławińska-Czarnak, J. (2015) Comparison of Infectious Agents Susceptibility to Photocatalytic Effects of Nanosized Titanium and Zinc Oxides: A Practical Approach. Nanoscale Research Letters. [Online] 10. Available from: doi:10.1186/s11671-015-1023-z.

Borkow, G. \& Gabbay, J. (2008) Biocidal textiles can help fight nosocomial infections. Medical Hypotheses. [Online] 70 (5), 990-994. Available from: doi:10.1016/j.mehy.2007.08.025.

Boyce, J.M. (2007) Environmental contamination makes an important contribution to hospital infection. The Journal of Hospital Infection. [Online] 65 Suppl 2, 50-54. Available from: doi:10.1016/S01956701(07)60015-2.

Brown, A.N., Smith, K., Samuels, T.A., Lu, J., et al. (2012) Nanoparticles functionalized with ampicillin destroy multiple-antibiotic-resistant isolates of Pseudomonas aeruginosa and Enterobacter aerogenes and methicillin-resistant Staphylococcus aureus. Applied and Environmental Microbiology. [Online] 78 (8), 2768-2774. Available from: doi:10.1128/ AEM.06513-11.

Callaghan, I. (1998) Bacterial contamination of nurses' uniforms: a study. Nursing Standard (Royal College of Nursing (Great Britain): 1987). [Online] 13 (1), 3742. Available from: doi:10.7748/ns1998.09.13.1.37. c2525.

Carré, G., Hamon, E., Ennahar, S., Estner, M., et al. (2014) TiO2 Photocatalysis Damages Lipids and Proteins in Escherichia coli. Applied and Environmental Microbiology. [Online] 80 (8), 2573-2581. Available from: doi:10.1128/AEM.03995-13.

Cataño, O, J.C., Echeverri, L.M., Szela, C., et al. (2012) Bacterial Contamination of Clothes and Environmental Items in a Third-Level Hospital in Colombia, Bacterial Contamination of Clothes and Environmental Items in a Third-Level Hospital in Colombia. Interdisciplinary Perspectives on Infectious Diseases, Interdisciplinary Perspectives on Infectious Diseases. [Online] 2012, 2012, e507640. Available from: doi:10.1155/2012/507640, 10.1155/2012/507640.

Cui, H., Jiang, J., Gu, W., Sun, C., et al. (2010) Photocatalytic Inactivation Efficiency of Anatase Nano-TiO2 Sol on the H9N2 Avian Influenza Virus. Photochemistry and Photobiology. [Online] 86 (5), 1135-1139. Available from: doi:10.1111/j.1751-1097.2010.00763.x.

Cui, Y., Zhao, Y., Tian, Y., Zhang, W., et al. (2012) The molecular mechanism of action of bactericidal gold nanoparticles on Escherichia coli. Biomaterials. [Online] 33 (7), 2327-2333. Available from: doi:10.1016/j.biomaterials.2011.11.057.

Damani, N. (2012) Manual of Infection Prevention and Control. 3 edition. Oxford University Press.

Dastjerdi, R., Mojtahedi, M.R.M., Shoshtari, A.M. \& Khosroshahi, A. (2010) Investigating the production and properties of $\mathrm{Ag} / \mathrm{TiO} 2 / \mathrm{PP}$ antibacterial nanocomposite filament yarns. The Journal of The Textile Institute. [Online] 101 (3), 204-213. Available from: doi:10.1080/00405000802346388.

Delavari, M., Dalimi, A., Ghaffarifar, F. \& Sadraei, J. (2014) In Vitro Study on Cytotoxic Effects of ZnO Nanoparticles on Promastigote and Amastigote Forms of Leishmania major (MRHO/IR/75/ER). Iranian Journal of Parasitology. 9 (1), 6-13.

Dodd, A.C., McKinley, A.J., Saunders, M. \& Tsuzuki, T. (2006) Effect of Particle Size on the Photocatalytic Activity of Nanoparticulate Zinc Oxide. Journal of Nanoparticle Research. [Online] 8 (1), 43-51. Available from: doi:10.1007/s11051-005-5131-z.

Dohmae, S., Okubo, T., Higuchi, W., Takano, T., et al. (2008) Bacillus cereus nosocomial infection from reused towels in Japan. The Journal of Hospital Infection. [Online] 69 (4), 361-367. Available from: doi:10.1016/j.jhin.2008.04.014

Eremenko, A.M., Petrik, I.S., Smirnova, N.P., Rudenko, A.V., et al. (2016) Antibacterial and Antimycotic Activity of Cotton Fabrics, Impregnated with Silver and Binary Silver/Copper Nanoparticles. Nanoscale Research Letters. [Online] 11. Available from: doi:10.1186/ s11671-016-1240-0.

Esteves, D.C., Pereira, V.C., Souza, J.M., Keller, R., et al. (2016) Influence of biological fluids in bacterial viability on different hospital surfaces and fomites. American Journal of Infection Control. [Online] 44 (3), 311-314. Available from: doi:10.1016/j. ajic.2015.09.033.

Fijan, S. \& Turk, S.Š. (2012) Hospital textiles, are they a possible vehicle for healthcare-associated infections? International Journal of Environmental Re- 
search and Public Health. [Online] 9 (9), 3330-3343. Available from: doi:10.3390/ijerph9093330.

Fu, G., Vary, P.S. \& Lin, C.-T. (2005) Anatase TiO2 Nanocomposites for Antimicrobial Coatings. The Journal of Physical Chemistry B. [Online] 109 (18), 8889-8898. Available from: doi:10.1021/jp0502196.

Gaikwad, S., Ingle, A., Gade, A., Rai, M., et al. (2013) Antiviral activity of mycosynthesized silver nanoparticles against herpes simplex virus and human parainfluenza virus type 3. International Journal of Nanomedicine. [Online] 8, 4303-4314. Available from: doi:10.2147/IJN.S50070.

Gondal, M.A., Alzahrani, A.J., Randhawa, M.A. \& Siddiqui, M.N. (2012) Morphology and antifungal effect of nano-ZnO and nano-Pd-doped nano-ZnO against Aspergillus and Candida. Journal of Environmental Science and Health. Part A, Toxic/Hazardous Substances \& Environmental Engineering. [Online] 47 (10), 1413-1418. Available from: doi:10.1080/109 34529.2012 .672384 .

Hernández-Sierra, J.F., Ruiz, F., Pena, D.C.C., Martínez-Gutiérrez, F., et al. (2008) The antimicrobial sensitivity of Streptococcus mutans to nanoparticles of silver, zinc oxide, and gold. Nanomedicine: Nanotechnology, Biology, and Medicine. [Online] 4 (3), 237-240. Available from: doi:10.1016/j.nano.2008.04.005.

Huang, Z., Zheng, X., Yan, D., Yin, G., et al. (2008) Toxicological Effect of ZnO Nanoparticles Based on Bacteria. Langmuir. [Online] 24 (8), 4140-4144. Available from: doi:10.1021/la7035949.

Hwang, I., Lee, J., Hwang, J.H., Kim, K.-J., et al. (2012) Silver nanoparticles induce apoptotic cell death in Candida albicans through the increase of hydroxyl radicals. The FEBS journal. [Online] 279 (7), 1327-1338. Available from: doi:10.1111/j.17424658.2012.08527.x.

Jacobson, K.H., Gunsolus, I.L., Kuech, T.R., Troiano, J.M., et al. (2015) Lipopolysaccharide Density and Structure Govern the Extent and Distance of Nanoparticle Interaction with Actual and Model Bacterial Outer Membranes. Environmental Science \& Technology. [Online] 49 (17), 10642-10650. Available from: doi:10.1021/acs.est.5b01841.

Jiang, J.F., Cui, H.X., Yang, T., Cai, H.C., et al. (2009) Inactivation efficiency of nano-Cu2+/TiO2 on avian influenza (H9N2). Journal of Funtional Materials. 40, 1403-1406.
Kairyte, K., Kadys, A. \& Luksiene, Z. (2013) Antibacterial and antifungal activity of photoactivated $\mathrm{ZnO}$ nanoparticles in suspension. Journal of Photochemistry and Photobiology. B, Biology. [Online] 128, 78-84. Available from: doi:10.1016/j.jphotobiol.2013.07.017.

Karunakaran, C., Rajeswari, V. \& Gomathisankar, P. (2011) Enhanced photocatalytic and antibacterial activities of sol-gel synthesized $\mathrm{ZnO}$ and Ag-ZnO. Materials Science in Semiconductor Processing. [Online] 14 (2), 133-138. Available from: doi:10.1016/j. mssp.2011.01.017.

Kim, J.S., Kuk, E., Yu, K.N., Kim, J.-H., et al. (2007) Antimicrobial effects of silver nanoparticles. Nanomedicine: Nanotechnology, Biology and Medicine. [Online] 3 (1), 95-101. Available from: doi:10.1016/j. nano.2006.12.001.

Kim, K.-J., Sung, W.S., Moon, S.-K., Choi, J.-S., et al. (2008) Antifungal effect of silver nanoparticles on dermatophytes. Journal of Microbiology and Biotechnology. 18 (8), 1482-1484.

Kim, K.-J., Sung, W.S., Suh, B.K., Moon, S.-K., et al. (2009) Antifungal activity and mode of action of silver nano-particles on Candida albicans. Biometals: An International Journal on the Role of Metal Ions in Biology, Biochemistry, and Medicine. [Online] 22 (2), 235-242. Available from: doi:10.1007/s10534008-9159-2.

Klevens, R.M., Edwards, J.R., Richards, C.L., Horan, T.C., et al. (2007) Estimating health care-associated infections and deaths in U.S. hospitals, 2002. Public Health Reports (Washington, D.C.: 1974). 122 (2), 160-166.

Knetsch, M.L.W. \& Koole, L.H. (2011) New strategies in the development of antimicrobial coatings: The example of increasing usage of silver and silver nanoparticles. Polymers. [Online] 3 (1), 340-366. Available from: doi:10.3390/polym3010340.

Kotsanas, D., Wijesooriya, W.R.P.L.I., Sloane, T., Stuart, R.L., et al. (2014) The silver lining of disposable sporicidal privacy curtains in an intensive care unit. American Journal of Infection Control. [Online] 42 (4), 366-370. Available from: doi:10.1016/j. ajic.2013.11.013.

Lara, H.H., Ayala-Nuñez, N.V., Ixtepan-Turrent, L. \& Rodriguez-Padilla, C. (2010) Mode of antiviral action of silver nanoparticles against HIV-1. Journal of 
Nanobiotechnology. [Online] 8, 1. Available from: doi:10.1186/1477-3155-8-1.

Lara, H.H., Ayala-Núñez, N.V., Turrent, L. del C.I. \& Padilla, C.R. (2009) Bactericidal effect of silver nanoparticles against multidrug-resistant bacteria. World Journal of Microbiology and Biotechnology. [Online] 26 (4), 615-621. Available from: doi:10.1007/ s11274-009-0211-3.

Li, S., Zhu, T., Huang, J., Guo, Q., et al. (2017) Durable antibacterial and UV-protective Ag/TiO2@ fabrics for sustainable biomedical application. International Journal of Nanomedicine. [Online] 12, 2593-2606. Available from: doi:10.2147/IJN.S132035.

Ling, M.L., Apisarnthanarak, A., Thu, L.T.A., Villanueva, V., et al. (2015) APSIC Guidelines for environmental cleaning and decontamination. Antimicrobial Resistance and Infection Control. [Online] 4. Available from: doi:10.1186/s13756-015-0099-7.

Lok, C.-N., Ho, C.-M., Chen, R., He, Q.-Y., et al. (2007) Silver nanoparticles: partial oxidation and antibacterial activities. Journal of biological inorganic chemistry: JBIC: a publication of the Society of Biological Inorganic Chemistry. [Online] 12 (4), 527-534. Available from: doi:10.1007/s00775-007-0208-z.

Mishra, Y.K., Adelung, R., Röhl, C., Shukla, D., et al. (2011) Virostatic potential of micro-nano filopodia-like $\mathrm{ZnO}$ structures against herpes simplex virus-1. Antiviral Research. [Online] 92 (2), 305-312. Available from: doi:10.1016/j.antiviral.2011.08.017.

Montazer, M. \& Maali Amiri, M. (2014) ZnO nano reactor on textiles and polymers: ex situ and in situ synthesis, application, and characterization. The Journal of Physical Chemistry. B. [Online] 118 (6), 1453-1470. Available from: doi:10.1021/jp408532r.

Monteiro, D.R., Gorup, L.F., Silva, S., Negri, M., et al. (2011) Silver colloidal nanoparticles: antifungal effect against adhered cells and biofilms of Candida albicans and Candida glabrata. Biofouling. [Online] 27 (7), 711-719. Available from: doi:10.1080/089270 14.2011.599101.

Mu, H., Tang, J., Liu, Q., Sun, C., et al. (2016) Potent Antibacterial Nanoparticles against Biofilm and Intracellular Bacteria. Scientific Reports. [Online] 6, 18877. Available from: doi:10.1038/srep18877.

Oćwieja, M., Adamczyk, Z., Morga, M. \& Kubiak, K. (2015) Silver particle monolayers - Formation, stability, applications. Advances in Colloid and Interface
Science. [Online] 222, 530-563. Available from: doi:10.1016/j.cis.2014.07.001.

Ohl, M., Schweizer, M., Graham, M., Heilmann, K., et al. (2012) Hospital privacy curtains are frequently and rapidly contaminated with potentially pathogenic bacteria. American Journal of Infection Control. [Online] 40 (10), 904-906. Available from: doi:10.1016/j.ajic.2011.12.017.

OMS (n.d.) Carga mundial de infecciones asociadas a la atención sanitaria. [Online]. WHO. Available from: http://www.who.int/gpsc/country_work/burden_ hcai/es/ [Accessed: 13 January 2016].

Padmavathy, N. \& Vijayaraghavan, R. (2008) Enhanced bioactivity of $\mathrm{ZnO}$ nanoparticles-an antimicrobial study. Science and Technology of Advanced Materials. [Online] 9 (3), 35004. Available from: doi:10.1088/1468-6996/9/3/035004.

PAHO (2012) Vigilancia epidemiológica de las infecciones asociadas a la atención en salud. [Online]. p.54. Available from: http://www.paho.org/ hq/index.php?option=com_docman\&task=doc view\&gid=19272\&Itemid=.

Pal, S., Tak, Y.K. \& Song, J.M. (2007) Does the antibacterial activity of silver nanoparticles depend on the shape of the nanoparticle? A study of the gram-negative bacterium Escherichia coli. Applied and Environmental Microbiology. [Online] 73 (6), 1712-1720. Available from: doi:10.1128/AEM.02218-06.

Palza, H. (2015) Antimicrobial Polymers with Metal Nanoparticles. International Journal of Molecular Sciences. [Online] 16 (1), 2099-2116. Available from: doi:10.3390/ijms16012099.

Panácek, A., Kolár, M., Vecerová, R., Prucek, R., et al. (2009) Antifungal activity of silver nanoparticles against Candida spp. Biomaterials. [Online] 30 (31), 63336340. Available from: doi:10.1016/j.biomaterials.2009.07.065.

Panagea, S., Winstanley, C., Walshaw, M.J., Ledson, M.J., et al. (2005) Environmental contamination with an epidemic strain of Pseudomonas aeruginosa in a Liverpool cystic fibrosis centre, and study of its survival on dry surfaces. The Journal of Hospital Infection. [Online] 59 (2), 102-107. Available from: doi:10.1016/j.jhin.2004.09.018.

Perelshtein, I., Applerot, G., Perkas, N., Grinblat, J., et al. (2012) A one-step process for the antimicrobial finishing of textiles with crystalline $\mathrm{TiO} 2$ nano- 
particles. Chemistry (Weinheim an Der Bergstrasse, Germany). [Online] 18 (15), 4575-4582. Available from: doi:10.1002/chem.201101683.

Perera, S., Bhushan, B., Bandara, R., Rajapakse, G., et al. (2013) Morphological, antimicrobial, durability, and physical properties of untreated and treated textiles using silver-nanoparticles. Colloids and Surfaces A: Physicochemical and Engineering Aspects. [Online] 436, 975-989. Available from: doi:10.1016/j.colsurfa.2013.08.038.

Perry, C., Marshall, R. \& Jones, E. (2001) Bacterial contamination of uniforms. The Journal of Hospital Infection. [Online] 48 (3), 238-241. Available from: doi:10.1053/jhin.2001.0962.

Petkova, P., Francesko, A., Perelshtein, I., Gedanken, A., et al. (2016) Simultaneous sonochemical-enzymatic coating of medical textiles with antibacterial $\mathrm{ZnO}$ nanoparticles. Ultrasonics Sonochemistry. [Online] 29, 244-250. Available from: doi:10.1016/j.ultsonch.2015.09.021.

Pollini, M., Russo, M., Licciulli, A., Sannino, A., et al. (2009) Characterization of antibacterial silver coated yarns. Journal of Materials Science. Materials in Medicine. [Online] 20 (11), 2361-2366. Available from: doi:10.1007/s10856-009-3796-z.

Rahimi, M.T., Ahmadpour, E., Rahimi Esboei, B., Spotin, A., et al. (2015) Scolicidal activity of biosynthesized silver nanoparticles against Echinococcus granulosus protoscolices. International Journal of Surgery. [Online] 19, 128-133. Available from: doi:10.1016/j. ijsu.2015.05.043.

Ramani, M., Ponnusamy, S., Muthamizhchelvan, C., Cullen, J., et al. (2013) Morphology-directed synthesis of $\mathrm{ZnO}$ nanostructures and their antibacterial activity. Colloids and Surfaces. B, Biointerfaces. [Online] 105, 24-30. Available from: doi:10.1016/j.colsurfb.2012.12.056.

Ramasamy, M., Lee, J.-H. \& Lee, J. (2016) Potent antimicrobial and antibiofilm activities of bacteriogenically synthesized gold-silver nanoparticles against pathogenic bacteria and their physiochemical characterizations. Journal of Biomaterials Applications. [Online] 31 (3), 366-378. Available from: doi:10.1177/0885328216646910.

Reddy, L.S., Nisha, M.M., Joice, M. \& Shilpa, P.N. (2014) Antimicrobial activity of zinc oxide ( $\mathrm{ZnO}$ ) nanoparticle against Klebsiella pneumoniae. Pharmaceutical Bio- logy. [Online] 52 (11), 1388-1397. Available from: doi:10.3109/13880209.2014.893001.

Saini, P., Saha, S.K., Roy, P., Chowdhury, P., et al. (2016) Evidence of reactive oxygen species (ROS) mediated apoptosis in Setaria cervi induced by green silver nanoparticles from Acacia auriculiformis at a very low dose. Experimental Parasitology. [Online] 160, 39-48. Available from: doi:10.1016/j.exppara.2015.11.004.

Sarkar, S., Guibal, E., Quignard, F. \& SenGupta, A.K. (2012) Polymer-supported metals and metal oxide nanoparticles: synthesis, characterization, and applications. Journal of Nanoparticle Research. [Online] 14 (2), 1-24. Available from: doi:10.1007/s11051011-0715-2.

Sasahara, T., Hayashi, S., Morisawa, Y., Sakihama, T., et al. (2011) Bacillus cereus bacteremia outbreak due to contaminated hospital linens. European Journal of Clinical Microbiology \& Infectious Diseases: Official Publication of the European Society of Clinical Microbiology. [Online] 30 (2), 219-226. Available from: doi:10.1007/s10096-010-1072-2.

Sattar, S.A., Springthorpe, S., Mani, S., Gallant, M., et al. (2001) Transfer of bacteria from fabrics to hands and other fabrics: development and application of a quantitative method using Staphylococcus aureus as a model. Journal of Applied Microbiology. 90 (6), 962-970.

Schweizer, M., Graham, M., Ohl, M., Heilmann, K., et al. (2012) Novel hospital curtains with antimicrobial properties: a randomized, controlled trial. Infection Control and Hospital Epidemiology. [Online] 33 (11), 1081-1085. Available from: doi:10.1086/668022.

Sehulster, L.M., Chinn, R.Y.W., Arduino, M.J., Carpenter, J., et al. (2004) Guidelines for environmental infection control in health-care facilities. Recommendations from CDC and the Healthcare Infection Control Practices Advisory Committee (HICPAC).p.235.

Sexton, T., Clarke, P., O’Neill, E., Dillane, T., et al. (2006) Environmental reservoirs of methicillin-resistant Staphylococcus aureus in isolation rooms: correlation with patient isolates and implications for hospital hygiene. The Journal of Hospital Infection. [Online] 62 (2), 187-194. Available from: doi:10.1016/j. jhin.2005.07.017.

Shaheen, T.I., El-Naggar, M.E., Abdelgawad, A.M. \& Hebeish, A. (2016) Durable antibacterial and UV 
protections of in situ synthesized zinc oxide nanoparticles onto cotton fabrics. International Journal of Biological Macromolecules. [Online] 83 (Supplement C), 426-432. Available from: doi:10.1016/j. ijbiomac.2015.11.003.

Shahid-ul-Islam, Butola, B.S. \& Mohammad, F. (2016) Silver nanomaterials as future colorants and potential antimicrobial agents for natural and synthetic textile materials. RSC Advances. [Online] 6 (50), 4423244247. Available from: doi:10.1039/C6RA05799C.

Shamaila, S., Zafar, N., Riaz, S., Sharif, R., et al. (2016) Gold Nanoparticles: An Efficient Antimicrobial Agent against Enteric Bacterial Human Pathogen. Nanomaterials (Basel, Switzerland). [Online] 6 (4). Available from: doi:10.3390/nano6040071.

Sharma, D., Rajput, J., Kaith, B.S., Kaur, M., et al. (2010) Synthesis of $\mathrm{ZnO}$ nanoparticles and study of their antibacterial and antifungal properties. Thin Solid Films. [Online] 519 (3), 1224-1229. Available from: doi:10.1016/j.tsf.2010.08.073.

Sreelakshmi, C., Datta, K.K.R., Yadav, J.S. \& Reddy, B.V.S. (2011) Honey derivatized Au and Ag nanoparticles and evaluation of its antimicrobial activity. Journal of Nanoscience and Nanotechnology. 11 (8), 69957000 .

Thabet, S., Simonet, F., Lemaire, M., Guillard, C., et al. (2014) Impact of photocatalysis on fungal cells: depiction of cellular and molecular effects on Saccharomyces cerevisiae. Applied and Environmental Microbiology. [Online] 80 (24), 7527-7535. Available from: doi:10.1128/AEM.02416-14.

Tran Thi, V.H. \& Lee, B.-K. (2017) Development of multifunctional self-cleaning and UV blocking cotton fabric with modification of photoactive $\mathrm{ZnO}$ coating via microwave method. Journal of Photochemistry and Photobiology A: Chemistry. [Online] 338 (Supplement C), 13-22. Available from: doi:10.1016/j.jphotochem.2017.01.020.

Treakle, A.M., Thom, K.A., Furuno, J.P., Strauss, S.M., et al. (2009) Bacterial contamination of health care workers' white coats. American Journal of Infection Control. [Online] 37 (2), 101-105. Available from: doi:10.1016/j.ajic.2008.03.009.

Trillis, F., Eckstein, E.C., Budavich, R., Pultz, M.J., et al. (2008) Contamination of hospital curtains with healthcare-associated pathogens. Infection Control and Hospital Epidemiology. [Online] 29 (11), 10741076. Available from: doi:10.1086/591863.

Uğur, S.S., Sarışık, M., Aktaş, A.H., Uçar, M.C., et al. (2010) Modifying of Cotton Fabric Surface with Nano-ZnO Multilayer Films by Layer-by-Layer Deposition Method. Nanoscale Research Letters. [Online] 5 (7), 1204-1210. Available from: doi:10.1007/s11671010-9627-9.

Valderrama Beltran, S.L., Ariza Ayala, B.E. \& Osorio Pinzon, J.V. (2010) Medio ambiente e infección. In: Infecciones Hospitalarias. 3rd edition. Bogota, Editorial Medica Internacional. pp. 126-147.

Vazquez-Muñoz, R., Avalos-Borja, M. \& Castro-Longoria, E. (2014) Ultrastructural Analysis of Candida albicans When Exposed to Silver Nanoparticles. PLOS ONE. [Online] 9 (10). Available from: doi:10.1371/journal.pone.0108876 [Accessed: 2 March 2017].

Wani, I.A., Ahmad, T. \& Manzoor, N. (2013) Size and shape dependant antifungal activity of gold nanoparticles: a case study of Candida. Colloids and Surfaces. B, Biointerfaces. [Online] 101, 162-170. Available from: doi:10.1016/j.colsurfb.2012.06.005.

WHO (2011) Report on the burden of health care-associated infection worldwide. [Online]. p.40. Available from: http://www.who.int/gpsc/country_work/ burden_hcai/en/ [Accessed: 13 January 2016].

Wiener-Well, Y., Galuty, M., Rudensky, B., Schlesinger, Y., et al. (2011) Nursing and physician attire as possible source of nosocomial infections. American Journal of Infection Control. [Online] 39 (7), 555-559. Available from: doi:10.1016/j.ajic.2010.12.016.

Windler, L., Height, M. \& Nowack, B. (2013) Comparative evaluation of antimicrobials for textile applications. Environment International. [Online] 53, 62-73. Available from: doi:10.1016/j.envint.2012.12.010.

Xiang, D., Zheng, Y., Duan, W., Li, X., et al. (2013) Inhibition of A/Human/Hubei/3/2005 (H3N2) influenza virus infection by silver nanoparticles in vitro and in vivo. International Journal of Nanomedicine. [Online] 8, 4103-4114. Available from: doi:10.2147/ IJN.S53622.

Xiao, G., Zhang, X., Zhao, Y., Su, H., et al. (2014) The behavior of active bactericidal and antifungal coating under visible light irradiation. Applied Surface Science. [Online] 292, 756-763. Available from: doi:10.1016/j.apsusc.2013.12.044. 
Xie, Y., He, Y., Irwin, P.L., Jin, T., et al. (2011) Antibacterial activity and mechanism of action of zinc oxide nanoparticles against Campylobacter jejuni. Applied and Environmental Microbiology. [Online] 77 (7), 2325-2331. Available from: doi:10.1128/ AEM.02149-10.

Zarrindokht Emami-Karvani, P.C. (2012) Antibacterial activity of $\mathrm{ZnO}$ nanoparticle on Gram-positive and Gram-negative bacteria. African Journal of Microbiology Research. [Online] 5 (18). Available from: doi:10.5897/AJMR10.159.

Zhang, L., Ding, Y., Povey, M. \& York, D. (2008) ZnO nanofluids - A potential antibacterial agent. Progress in Natural Science. [Online] 18 (8), 939-944. Available from: doi:10.1016/j.pnsc.2008.01.026.

Zhang, Y., Shareena Dasari, T.P., Deng, H. \& Yu, H. (2015) Antimicrobial Activity of Gold Nanoparticles and Ionic Gold. Journal of Environmental Science and Health. Part C, Environmental Carcinogenesis \& Ecotoxicology Reviews. [Online] 33 (3), 286-327. Available from: doi:10.1080/10590501.2015.1055161.

\section{PARA CITAR ESTE ARTÍCULO / TO REFERENCE THIS ARTICLE / PARA CITAR ESTE ARTIGO /}

Zapata-Giraldo, J.; Botero, L.E.; Mejía, M.L.; Escobar-Mora, N.; Ortiz-Trujillo, I.; Galeano U., B.J.; Hoyos, L.M.; Cuesta, D. (2018). Textiles funcionales como barrera de protección ante infecciones asociadas a la atención en salud. Revista EIA, 15(29), enero-junio, pp. 13-29. [Online]. Disponible en: https://doi.org/10.24050/reia.v15i29.1166 